\title{
Saúde mental, suporte familiar e adesão ao tratamento: associações no contexto HIV/Aids
}

\author{
Luiza Ažem Camargo - Instituto de Infectologia Emílio Ribas, São Paulo, Brasil \\ Cláudio Garcia Capitão - Universidade São Francisco, Itatiba, Brasil \\ Elvira Maria Ventura Filipe - Coordenadoria de Controle de Doenças da Secretaria de Estado da Saúde de São Paulo, São Paulo, Brasil
}

\begin{abstract}
Resumo
A adesão dos pacientes ao tratamento antirretroviral tem sido um dos maiores desafios no contexto HIV/Aids. São muitos os fatores implicados, e este estudo buscou privilegiar os relativos à saúde mental e suporte familiar, investigando sua associação com a adesão. Para tanto, foram utilizados um questionário semiestruturado composto de informações sociodemográficas, clínicas e adesão, e os instrumentos Questionário de Saúde Geral de Goldberg (QSG) e Inventário de Percepção de Suporte Familiar (IPSF). A amostra foi composta por 73 pacientes com HIV/Aids, com idades entre 29 e 67 anos, em tratamento antirretroviral em um ambulatório de São Paulo. Os resultados apontaram índices significativos de adesão irregular aos antirretrovirais e de perfis sintomáticos para transtornos mentais nessa população. Foram encontradas correlações significativas entre adesão e o fator "desejo de morte" do QSG, e entre adesão e quase todos os fatores do IPSF, evidenciando a importância desses fatores na adesão ao tratamento.

Palavras-chave: Adesão à medicação; Antirretrovirais; Família; Saúde mental; HIV/Aids.
\end{abstract}

\section{Mental health, family support and treatment adherence: associations in the context of HIV / AIDS}

\begin{abstract}
The patient's adherence to the antiretroviral drug therapy has been one of the biggest challenges in the HIV/Aids context. Among the many implicated factors, this study sought to study the factors related to mental disorders and family support, investigating their association with the adhesion. For that matter, a semi-structured questionnaire composed by social-demographic, clinical and adherence information was applied. The Goldberg General Health Questionnaire (GHQ) and the Perceived Family Support Inventory (PFSI) were also used. The sample was composed by $73 \mathrm{HIV} /$ Aids patients in antiretroviral therapy, aged between 29 and 67 years old on health service in São Paulo. The results point at significant indicators of irregular adherence to the antiretroviral therapy and of symptomatic profiles of mental disorders in this population. A considerable correlation between adherence and the "death wish" factor of GHQ were found, and also between adherence and almost all the PFSI factors, demonstrating the importance of these factors in therapy adherence.
\end{abstract}

Keywords: Medication adherence; Antiretroviral; Family; Mental health; HIV/Aids.

Salud mental, soporte familiar y adhesión al tratamiento: asociaciones en el contexto VIH/Sida

\begin{abstract}
Resumen
La adhesión de los pacientes al tratamiento antirretroviral es uno de los mayores desafíos en el contexto VIH/Sida. En este estudio han sido privilegiados e investigados los factores relativos a la salud mental y soporte familiar. Para esto, se aplicó un cuestionario semiestructurado compuesto por informaciones sociodemográficas, clínicas y de adhesión, y los instrumentos Cuestionario de Salud General de Goldberg (QSG) e Inventario de Percepción de Soporte Familiar (IPSF). La muestra estuvo compuesta por 73 pacientes con VIH/Sida, entre 29 y 67 años, en tratamiento antirretroviral en un ambulatorio de São Paulo. Los resultados mostraron indicios significativos de adhesión irregular a los antirretrovirales y de perfiles sintomáticos para trastornos mentales en esta población. Se encontraron correlaciones significativas entre adhesión y el factor "deseo de muerte" del QSG, y entre adhesión y prácticamente todos los factores del IPSF, mostrando la importancia de estos factores en la adhesión al tratamiento.

Palabras-clave: Adhesión a la medicación; Antirretrovirales; Familia; Salud mental; VIH/Sida.
\end{abstract}

\section{Introdução}

$\mathrm{O}$ alto índice de morbidade e mortalidade torna a síndrome da imunodeficiência adquirida (Aids), desde o aparecimento do primeiro caso no início da década de 1980, um urgente problema de saúde pública, e, desde então, diversos desafios vêm sendo colocados à humanidade (Gir, Vaichulonis \& Oliveira, 2005). O perfil epidemiológico e as tendências iniciais de infecção e transmissão foram se modificando ao longo dos anos. A ideia da existência de grupos de risco específicos

Disponivel em www.scielo.br (ex: homossexuais e usuários de drogas injetáveis) deu lugar à ideia de comportamentos de risco (ex: sexo desprotegido) e, posteriormente, à de vulnerabilidade. A disseminação entre heterossexuais passa a ser evidente, e esta é hoje a subcategoria de exposição sexual com maior número de casos notificados (Brasil, 2007; Dourado, Veras, Barreira \& Brito, 2006; Moraes, Oliveira \& Tostes, 2006).

No que se refere aos altos índices de morbidade e mortalidade, houve grande queda com a introdução de potentes esquemas antirretrovirais (ARV) na prática 
clínica (Rachid \& Schechter, 2005). Os primeiros medicamentos antirretrovirais começaram a ser pesquisados ainda na década de 1980, mas os benefícios que proporcionavam ao paciente eram temporários. A partir de 1996, com o advento de novas classes de ARV, iniciou-se a utilização da terapia ARV combinada (bighly active antiretroviraltherapy - HAART) e passou-se, então, à obtenção de êxitos significativos no tratamento da infecção pelo vírus da imunodeficiência humana (HIV) (Seidl, Melchiades, Farias \& Brito, 2007).

A HAART proporcionou condições para que a aids passasse a ser considerada uma doença crônica, e não mais uma sentença de morte. Essa terapia medicamentosa interfere na multiplicação do HIV no organismo e, com isso, na progressão da doença para aids. Como consequência do uso adequado da medicação ARV, é esperado um aumento ou melhora da imunidade (linfócitos CD4) e uma diminuição do valor de carga viral do HIV, que pode chegar a ser indetectável.Com a distribuição universal dos medicamentos ARV, ou seja, a distribuição gratuita para todos os indivíduos que necessitam do tratamento, prolongou-se a sobrevida e uma melhora na qualidade de vida das pessoas vivendo com o HIV no Brasil (Gir \& cols., 2005). Atualmente pode-se dizer que diversas problemáticas referentes à epidemia foram superadas, mas ainda existem desafios, os quais podem ser observados, por exemplo, por meio dos Boletins do Ministério da Saúde e da United Nations Programmeon HIV/Aids (Brasil, 2011; UNAIDS, 2010).

Das cerca de 15 milhões de pessoas vivendo com HIV e aids nos países considerados de baixa e média renda e que necessitam de tratamento, por volta de 5,2 milhões já têm acesso à TARV, o que pode significar a ocorrência de menos mortes relacionadas à epidemia, embora essas cinco milhões de pessoas não representem mais do que 35\% do total. Nas Américas Central e do Sul registraram-se cerca de 92.000 novos casos e 58.000 mortes relacionadas à HIV/Aids no ano de 2009. Estima-se que aproximadamente 1,4 milhões de pessoas vivam com HIV/Aids nesses países, sendo em torno de 36.000 crianças. Cerca de um terço dessas pessoas vivem no Brasil, onde os esforços de prevenção precoce e tratamento têm sido considerados de grande importância para a manutenção da prevalência de HIV abaixo de $1 \%$ em adultos, durante toda a última década (UNAIDS, 2010). Ainda assim, no ano de 2010, 11.965 pessoas morreram de causas relacionadas à aids no Brasil (Brasil, 2011).

Os números continuam crescendo e o panorama da epidemia nas Américas Central e do Sul tem mudado pouco, pois uma parcela significativa dos casos concentra-se nas redes de homens que fazem sexo com homens (HSH), cuja prevalência nessa população, em 12 países da América Latina, gira em torno de 10\% (UNAIDS, 2010). Ao encontro desse dado observa-se, por exemplo, que no Brasil a prevalência da infecção pelo HIV na população HSH jovem, de 17 a 22 anos, passou de $0,56 \%$ em 2002, para 1,2\% em 2007. Entre homens de faixa etária de 15 a 24 anos, por sua vez, houve aumento proporcional da categoria $\mathrm{HSH}$, passando de $31,8 \%$ das infecções registradas em 1998, para 46,4\% em 2010, com um aumento da proporção de casos nas regiões Sudeste, Sul e Centro-Oeste, diminuição no Nordeste e estabilização na região Norte (Brasil, 2011).

Portanto, pode-se observar que, apesar de tantos ganhos, ainda existem muitos desafios em termos de prevenção, conscientização e acessibilidade ao tratamento a serem superados. Paralelo a isso, destacam-se também os desafios quanto aos direitos humanos. Sabe-se que o estigma, desde o início da epidemia, destacou-se como um dos maiores problemas a serem superados e que repercute em muitas esferas da vida das pessoas com HIV e aids. A UNAIDS (2010) considera ainda que o estigma discriminação e violência contra pessoas transgênero e homens que fazem sexo com homens aumenta o risco de infecção pelo HIV não só para essa população, mas também para seus/ suas respectivos (as) parceiros (as).

Outro desafio diz respeito à saúde mental, esfera de conhecimentos e práticas que, desde o aparecimento dos primeiros casos de aids na década de 1980 , verificouse diante de uma complexidade ímpar de fenômenos e sintomas associados. Algumas síndromes psiquiátricas clássicas, como os transtornos de humor, e particularmente a depressão, são frequentemente associadas ao HIV. Tal associação evidencia-se no fato de a prevalência desses transtornos na população infectada pelo HIV ser maior do que na população em geral (Moraes \& cols., 2006). A incidência de transtorno depressivo entre pacientes HIV, por exemplo, é a complicação psiquiátrica descrita com mais frequência, podendo variar de 4 a 40\% (Sadock \& Sadock, 2007; Straub, 2005).

Um estudo realizado por dois psiquiatras de um grande hospital escola de São Paulo, por exemplo, que visava avaliar a prevalência de depressão maior e outros transtornos depressivos em 120 mulheres infectadas pelo HIV, assim como comparar a prevalência entre pacientes assintomáticas e sintomáticas para HIV, obteve $25,8 \%$ de diagnósticos para depressão maior, sendo 13,3\% nas pacientes assintomáticas e 38,3\% 
nas sintomáticas. Vale ressaltar que ao menos $48 \%$ da amostra já havia experimentado, no mínimo, um episódio de depressão maior, antes ou depois do diagnóstico de HIV (Mello \& Malbergier, 2006).

Dentre os sintomas frequentemente referidos nas sintomatologias moderada e grave para depressão, observa-se a ideação suicida ou pensamento sobre morte. Nos pacientes com HIV (portadores do vírus, assintomáticos) e aids (sintomáticos, que já manifestaram alguma doença oportunista em decorrência da ação do HIV) encontram-se estudos avaliando particularmente essa sintomatologia. Um desses estudos visava investigar a prevalência e preditores de ideação e tentativas passadas de suicídio em homens homo e bissexuais, soropositivos $(n=164)$ ou não $(n=65)$ para HIV. Os pacientes sintomáticos para HIV $(\mathrm{n}=85)$ apresentaram níveis mais altos de ideação que os assintomáticos $(n=79)$ e os soronegativos; foram encontrados altos níveis de tentativa passada tanto em soronegativos $(29 \%)$ quanto soropositivos (21\%). Dentre os fatores preditivos associados destacaram-se histórico de transtorno psiquiátrico (particularmente depressão), de uso de drogas e suicídio na família (Brian \& cols., 1998).

Outros diagnósticos ou sintomas que também merecem destaque nos pacientes com HIV e aids são os transtornos de adaptação ou ajustamento ( $5 \%$ a $20 \%$ ), insônia, dependência ou uso abusivo de álcool e outras drogas e, em menor número, os transtornos psicóticos (Sadock \& Sadock, 2007; Straub, 2005). A associação entre transtornos psiquiátricos e a infecção pelo HIV pode estar relacionada ao maior risco de exposição ao HIV, acarretado por alguns transtornos psiquiátricos; às circunstâncias de vida frequentemente associadas ao diagnóstico do HIV, como perda de emprego, abandono ou desestruturação familiar; ou mesmo à reação ao diagnóstico, como culpa ou medo da dor e da morte. Por outro lado, os transtornos psiquiátricos podem ser resultantes da própria infecção pelo HIV, pela ação do mesmo no sistema nervoso central (SNC), pela ocorrência de doenças oportunistas ou mesmo pela ação de medicamentos utilizados no tratamento (Moraes \& cols., 2006).

Nos casos de transtornos psiquiátricos resultantes da própria infecção pelo HIV, Sadock e Sadock (2007) e Straub (2005), analisando as características clínicas da aids, constataram a existência de fatores não neurológicos e neurológicos no desenvolvimento destes. Os fatores não neurológicos dizem respeito aos sintomas da própria infecção pelo HIV, às complicações causadas pelas doenças oportunistas que aparecem em decorrência da evolução da doença, que podem apresentar sinais e sintomas bastante semelhantes aos apresentados por alguns transtornos mentais do eixo I, por exemplo. A importância de tais complicações reside no fato de implicarem em efeitos biológicos para as funções cerebrais do paciente, assim como em efeitos psicológicos nos estados de humor e de ansiedade.

Entre os fatores neurológicos responsáveis pelo desenvolvimento de síndromes psiquiátricas associadas ao HIV encontra-se o transtorno neurocognitivo leve devido ao HIV e à demência associada. Essa última é uma condição do tipo cortical ou subcortical e pode afetar cerca de $50 \%$ dos pacientes em algum grau. No entanto, ao se avaliar um paciente com sintomas neuropsiquiátricos, é preciso atentar para o fato de que outras causas produzem sintomas semelhantes aos da demência e devem ser consideradas, como, por exemplo, infecções e neoplasias do SNC, decorrentes de doenças sistêmicas (oportunistas) e endocrinopatias, assim como respostas adversas do SNC a medicamentos (Lewi \& cols., 2007; Sadock \& Sadock, 2007; Straub, 2005).

Atualmente, no contexto HIV/Aids, um dos maiores desafios diz respeito à adesão dos pacientes à TARV. Segundo diretrizes do Ministério da Saúde uma boa adesão ao tratamento significa tomar corretamente os medicamentos antirretrovirais, seguir as doses corretas pelo tempo preestabelecido, bem como aderir ao serviço de saúde responsável (Brasil, 2007). Essa recomendação tem implícita uma noção de obediência e de que as medicações prescritas podem não ter sido fruto de uma decisão compartilhada. Por isso, em manual publicado em 2008 sobre condutas de adesão, o Ministério da Saúde frisa que a adesão é um processo colaborativo, de negociação entre usuário e profissionais de saúde e que visa fortalecer a autonomia para o autocuidado (Brasil, 2008).

A complexidade desse conceito pode ser facilmente percebida, na medida em que se constata que os índices de adesão, em grande parte dos estudos atuais, não são satisfatórios ou adequados para uma parcela significativa dos usuários. Enquanto alguns estudos apontam uma taxa média de adesão de $50 \%$ para todos os tipos de tratamento (Jordan, Lopes, Okazaki, Komatsu \& Nemes, 2000), outros mostram que a adesão à terapêutica ARV pode variar entre 40 e $80 \%$ (Ligani Júnior, Greco \& Carneiro, 2001). O estudo de Bonolo, Gomes e Guimarães (2007), que teve por objetivo apresentar uma revisão da literatura sobre adesão ao tratamento, refere uma prevalência média de nãoadesão de 
30,4\%, ou seja, em média, cerca de $70 \%$ dos pacientes em tratamento apresentam índices de adesão considerados adequados.

Com relação ao índice considerado adequado, a maioria dos estudos estabelece um ponto de corte que varia de 80 a $100 \%$ de uso das drogas prescritas. No entanto, não existe um método padronizado para avaliação da adesão ao tratamento nem mesmo um nível de adesão definido como ideal. Atualmente são utilizadas algumas estratégias pelas quais a adesão é aferida de forma indireta (exames laboratoriais, por exemplo), sendo, portanto, imperfeita. Daí a recomendação de se associar mais de uma abordagem e de se considerar o método utilizado e o nível de definição da adesão no momento de avaliar os resultados (Bonolo \& cols., 2007; Caraciolo, 2007; Jordan \& cols., 2000).

Dentre os métodos de avaliação utilizados, merecem ser citadas as medidas subjetivas, baseadas em autorrelatos; e as objetivas, baseadas em contagens de exames laboratoriais (CD4, carga viral) ou de pílulas (Bonolo \& cols., 2007; Caraciolo, 2007; Weiss \& Bangsberg, 2008). Embora classicamente acredite-se que os autorrelatos tendam a superestimar as medidas de adesão e que não são fiéis, pois partem dos pacientes, eles podem ser bons indicadores quando bem conduzidos (Bonolo \& cols., 2007; Duong \& cols., 2001; Jordan \& cols., 2000). Outra medida bastante empregada são os registros da farmácia, que podem ser utilizados para monitorar as retiradas de medicações na farmácia e obter indícios de abandono de tratamento ou, mesmo, uso irregular das medicações, de acordo com a frequência de dispensação pela farmácia (Caraciolo, 2007).

Com relação aos motivos associados à adesão ou não adesão à TARV, pode-se dizer que são diversos e de diferentes naturezas. Observam-se na literatura fatores relacionados à pessoa sob tratamento, à doença, ao tratamento em si e ao serviço ou profissional de saúde, alguns dos quais explicitados a seguir (Adão \& Caraciolo, 2007).

- Fatores relacionados à pessoa sob tratamento: perfil socioeconômico, sexo, idade, renda, escolaridade, uso de drogas, aceitação e nível de conhecimento da doença, sintomas psicológicos ou transtornos psiquiátricos associados e percepção de suporte social ou material inadequados (Adão \& Caraciolo, 2007; Brasil, 2008; Jordan \& cols., 2000).

- Fatores relacionados à doença: falta de sintomas aparentes, cronicidade e a melhora de parâmetros laboratoriais (Adão \& Caraciolo, 2007; Brasil, 2008; Jordan $\&$ cols., 2000).
- Fatores relacionados ao tratamento: tempo, tipo e complexidade de regime terapêutico; efeitos colaterais adversos, caracterização da doença e objetivos do tratamento; e mudança de estilo de vida (Adão \& Caraciolo, 2007; Brasil, 2008; Jordan \& cols., 2000).

- Fatores relacionados ao serviço e profissional de saúde: relação médico-paciente, postura e linguagem do profissional, confiança na equipe de saúde, tempo de duração da consulta e aspectos de acessibilidade ao Serviço (Adão \& Caraciolo, 2007; Brasil, 2008; Jordan $\&$ cols., 2000).

Visando atender aos objetivos estabelecidos no presente estudo, foram privilegiados, dentro da temática da adesão, os estudos de evidências de condições associadas à saúde mental e percepção de suporte social e familiar. Nesse sentido, destacam-se fatores como estresse psíquico e a não adaptação da rotina ou estilo de vida com as medicações; assim como a ocorrência de depressão e a falta de suporte (ou percepção deste como inadequado) familiar ou social (Baptista, 2007; Bonolo \& cols., 2007; Brasil, 2008; Gordillo, Del Amo, Soriano \& González-Lahoz, 1999).

Pessoas com transtornos psiquiátricos em geral e/ ou abuso de drogas costumam apresentar níveis mais baixos de adesão. Sobre o uso de drogas, é fundamental distinguir o uso atual daquele praticado no passado. Com relação à saúde mental, a maioria das investigações tem focado na depressão, utilizando mais escalas de sintomas contínuos do que categorias diagnósticas de depressão propriamente ditas (Weiss \& Bangsberg, 2008).

Foi de fato encontrada, na literatura, associação entre não adesão e sintomas depressivos, na qual os pacientes com sintomas depressivos chegaram a ser considerados três vezes mais propensos que os sem sintomas depressivos à não adesão ao regime terapêutico (Ammassari \& cols., 2004). Chega-se a mencionar que, além de apresentarem alta prevalência de depressão, os pacientes com HIV/Aids que possuem tal transtorno podem melhorar seus índices de adesão após prescrição de antidepressivos, e atingir índices ainda mais altos de adesão, se aderentes também ao antidepressivo (Weiss \& Bangsberg, 2008; Yun, Maravi, Kobayashi, Barton \& Davidson, 2005).

Um estudo mais abrangente em termos de saúde mental buscou verificar a relação entre sintomas psicopatológicos, qualidade de vida e adesão à TARV em 125 pacientes de um hospital do Porto (Portugal) utilizando os instrumentos BSI (Inventário Breve de Sintomas), CEAT-VIH (Questionário de Avaliação de 
Adesão à TARV) e o WHOQOL-Bref (Questionário Breve de Qualidade de Vida da Organização Mundial de Saúde). Os resultados apontaram correlação negativa entre sintomas psicopatológicos e adesão $(\mathrm{p}<0,01)$, indicando maior frequência de sintomas psicopatológicos em níveis mais baixos de adesão. Também foram encontradas correlações positivas $(\mathrm{p}<0,01)$ entre adesão e todos os domínios de qualidade de vida, e correlação negativa entre esses domínios e sintomas psicopatológicos (Reis, Lencastrea, Guerra \& Remorb, 2009). Não foi encontrado outro estudo que avaliasse essas mesmas variáveis.

São poucos os estudos que aproximam saúde mental e adesão à TARV, e dos publicados disponíveis, a maioria parece ser de estudos estrangeiros (Ammassari \& cols., 2004; Duong \& cols., 2001; Gordillo, Del Amo, Soriano \& González-Lahoz, 1999; Reis, Lencastrea, Guerra \& Remorb, 2009; Weiss \& Bangsberg, 2008; Yun, Maravi, Kobayashi, Barton \& Davidson, 2005). Dentre os poucos estudos brasileiros encontrados buscando esta associação, um estudo realizado em Belo Horizonte, MG, partiu do pressuposto de que depressão e ansiedade são comuns entre pacientes com HIV e aids, e buscou investigar se de fato podem ser preditores de não adesão. A adesão foi autorreportada e a ansiedade e depressão mensuradas por meio da Escala Hospitalar de Ansiedade e Depressão (HAD). Dos 293 indivíduos avaliados em dois centros de referência, $51,5 \%$ e 40,6\% tinham sintomas de ansiedade e depressão, respectivamente, que iam de leves a severos. A prevalência de não adesão foi de $37,2 \%$ e os resultados apontaram risco elevado de não adesão para os pacientes com sintomas severos de ansiedade, sem nenhuma associação estatística entre adesão e depressão (Campos, Guimarães \& Remien, 2010).

No que se refere ao suporte social ou familiar, alguns estudos apontam este como fator que pode interferir tanto positiva quanto negativamente na adesão (Baptista, 2007; Bonolo \& cols., 2007; Gordillo \& cols., 1999; Oliveira \& Sommermann, 2008; Seidl \& cols., 2007; Teodoro, 2012). Uma pesquisa realizada no Rio Grande do Sul com 120 portadores de HIV/Aids, que visava investigar fatores biopsicossociais preditivos para adesão à TARV, encontrou altos índices referidos de adesão (apenas 4,2\% referiram baixa adesão), baixos escores de ansiedade (75,9\% entre mínimo e leve no BAI) e depressão $(69,2 \%$ entre mínimo e leve no $\mathrm{BDI})$, correlação negativa entre ansiedade e adesão $(r=-0,40, \mathrm{p}<0,001)$, e entre depressão e adesão ( $\mathrm{r}=$ $0,5 ; \mathrm{p}<0,001)$, correlação positiva entre suporte social, qualidade de vida e adesão à TARV $(r=0,4 ; \mathrm{p}<0,001)$, destacando a importância do suporte social como moderador do estresse em contextos relacionados ao processo saúde-doença (Calvetti, 2010).

Outro trabalho, também realizado no Rio Grande do Sul, com 63 pacientes com HIV/Aids, encontrou resultados semelhantes. Aqui se destacou, além da correlação positiva entre suporte social e adesão $(r=0,43$; $\mathrm{p}<0,001)$, o índice significativo do grau de adesão caracterizado como baixo ou insuficiente $(47,6 \%)$ e a correlação negativa entre depressão e adesão $(r=-0,43$; $\mathrm{p}<0,001)$ (Giovelli, 2009).

Tomando a adesão à TARV como manifestação de saúde e a não adesão como um problema, pretendeu-se investigar possíveis fatores associados a essas condutas do lugar de profissional de saúde mental, ou seja, privilegiando a investigação de indicativos e transtornos mentais e percepção de suporte familiar. $\mathrm{O}$ objetivo principal deste estudo foi investigar a associação entre indicativos de transtornos mentais, percepção de suporte familiar e adesão à TARV em pacientes com HIV e aids de um ambulatório de referência em HIV/ aids da cidade de São Paulo.

\section{Método}

O presente estudo segue uma abordagem quantitativa não experimental transversal, um delineamento correlacional (Cozby, 2003; Dancey \& Reidy, 2006). Foram entrevistados 73 pacientes com HIV/aids, de ambos os sexos, maiores de 18 anos, que tinham conhecimento do seu diagnóstico e que no momento da entrevista, estavam em tratamento ARV no ambulatório há pelo menos dois meses.

\section{Participantes}

Entre as características sociodemográficas da amostra estudada, destacam-se a predominância do sexo masculino, com $61,6 \%(\mathrm{n}=45)$; a idade variou de 29 a 67 anos $(M=44,7 ; D P=9,2) ; 50,7 \%(\mathrm{n}=37)$ naturais e $89 \%(\mathrm{n}=65)$ residentes da Capital ou Grande São Paulo; 45,2\% ( $\mathrm{n}=33)$ declararam-se brancos, 35,6\% $(n=26)$ pardos e 19,2\% $(n=14)$ negros. Com relação à orientação sexual, $68,5 \%(n=50)$ declararam-se heterossexuais, $24,7 \%(n=18)$ homossexuais e $6,8 \%(n=5)$ bissexuais. Com relação ao estado marital, se somarem-se as frequências relativas aos que se denominaram casados e amasiados, $43,8 \%(n=32)$ da amostra se relaciona atualmente com alguém. A escolaridade média referida foi de 9 anos de estudo $(D P=4,3)$. A renda 
familiar foi medida em número de salários mínimos e variou de 0 a $100(M=4,8 ; D P=11,6)$.

\section{Instrumentos}

\section{A) Informações sociodemográficas}

Roteiro elaborado por um dos pesquisadores, composto de questões como, por exemplo, sexo, idade, cor/raça, escolaridade, estado marital e renda familiar. Estas questões foram elaboradas pensando-se em fatores que têm sido considerados relevantes pelas revisões da literatura acerca da temática da adesão ao tratamento.

\section{B) Informações clínicas e sobre adesão}

Roteiro composto de questões como tempo de diagnóstico e tratamento para o HIV, forma de infecção, uso de drogas, número de doenças oportunistas (decorrentes da queda de imunidade provocada pela evolução da doença) e internações e outros tratamentos. Nesse momento, as informações sobre adesão foram aferidas por autorrelato, por meio de perguntas sobre quais medicamentos toma, quantas doses e quantos esquecimentos de tomadas ocorreram na última semana e no último mês.

C) Questionário de Saúde Geral de Goldberg - QSG (Pasquali, Gouveia, Andriola, Miranda,\& Ramos, 1996).

O QSG é um instrumento de avaliação psicológica, de uso restrito e privativo do psicólogo. Foi desenvolvido em 1972 por Goldberg, na Inglaterra, com o objetivo de identificar a severidade do distúrbio psiquiátrico do respondente. Em razão de sua eficácia na identificação de pessoas com transtornos mentais, o QSG foi traduzido e adaptado para a população brasileira em 1996. Foram realizados os estudos necessários para validação, e alguns itens foram alterados para melhor compreensão na língua portuguesa. Em suma, é um instrumento válido e preciso, recomendado para avaliação de transtornos mentais não desviantes da população.

O QSG é composto de 60 itens sobre sintomas psiquiátricos não psicóticos, apresentados em um folheto e respondidos em uma escala tipo likert. Trata-se de um instrumento cujos itens compõem e permitem interpretação dos fatores estresse psíquico, desejo de morte, desconfiança no próprio desempenho, distúrbios do sono, distúrbios psicossomáticos e saúde geral. É chamado perfil sintomático, o expresso por percentis maiores ou iguais a 90; são considerados limítrofes percentis entre 85 e 90; e não sintomáticos percentis menores que 85 .

D) Inventário de Percepção de Suporte Familiar - IPSF (Baptista, 2010)

Trata-se de um instrumento de uso exclusivo do psicólogo, que avalia a forma como o indivíduo percebe sua relação com a família. Os itens foram construídos com base em diversos instrumentos nacionais e internacionais e, ainda, por meio dos relatos de 100 estudantes de uma Faculdade de Psicologia do interior de São Paulo, que responderam a uma pergunta aberta sobre o que seria, em sua opinião, uma família ideal.

O IPSF demonstrou qualidades psicométricas muito boas, tanto em relação aos tipos de fidedignidade estudados quanto em procedimentos de validação e verificação de consistência interna entre os itens/questões. A versão final foi composta de 42 questões, sendo todas fechadas, respondidas em uma escala de três pontos, modelo likert de sentido e pontuação crescente. A análise fatorial extraiu a presença de três fatores: fator 1 - afetivo-consistente - com 21 itens, evidencia as relações afetivas positivas intrafamiliares; fator 2 - adaptação familiar - com um total de 13 itens que expressam sentimentos negativos em relação à família; e fator 3 - autonomia familiar - com 8 itens, demonstra a percepção de autonomia que o indivíduo tem de sua família.

E) Roteiro para busca de dados em prontuários e registros da farmácia

Elaborado para atender aos objetivos da pesquisa, foi preenchido em ocasião posterior à aplicação do questionário. Trata-se de um instrumento semiestruturado, composto de informações clínicas obtidas do prontuário médico do paciente (CD4, carga viral) e da farmácia (regularidade de retiradas de medicação da farmácia).

\section{Procedimentos}

Todos os participantes foram convidados a participar do estudo, na sala de espera e corredores do ambulatório, pelos próprios pesquisadores. Primeiramente, eram apresentados e verificados os critérios de inclusão, e em seguida, encaminhados a salas reservadas, onde os participantes recebiam as demais informações sobre a pesquisa, como objetivos, instrumentos a serem aplicados e tempo de duração da entrevista. O projeto de pesquisa foi devidamente aprovado pelo Comitê de Ética e todas as informações sobre a pesquisa constam no Termo de Consentimento Livre-esclarecido, que foi 
preenchido e assinado por todos que consentiram na participação, de acordo com os preceitos éticos que regem as pesquisas com seres humanos.

Com relação à análise dos dados, primeiramente foram feitas as estatísticas descritivas, em que se calcularam médias, valores mínimos e máximos e desvios padrão das variáveis numéricas, e as frequências das variáveis categóricas. Em seguida, foram realizadas correlações bivariadas de Pearson, visando descobrir se existia relacionamento significativo ou associação entre as variáveis investigadas, bem como a direção, força e magnitude desses relacionamentos. Foram realizadas também análises de regressão linear múltipla, visando verificar se e como variáveis de interesse aos objetivos do estudo podem ser consideradas explicativas ou preditoras de outras variáveis (dependentes ou de critério) (Dancey \& Reidy, 2006).

\section{Resultados e Discussão}

Sobre a forma de infecção pelo HIV, 87,7\% ( $n=64)$ referiram ter contraído o HIV por via sexual, sendo que destes, $56,2 \%(\mathrm{n}=41)$ por via heterossexual. A média de tempo de diagnóstico foi de 12 anos e de TARV foi de 10 anos. A adesão à TARV foi aferida por meio de autorrelato, registros de retirada de medicação da farmácia e dados clínicos de exames laboratoriais. Os autorrelatos foram acerca de esquecimentos de doses, cuja média referida na semana anterior à entrevista foi de 1 comprimido, e no último mês de 6 comprimidos esquecidos. Também foi perguntado sobre o esquema de TARV em uso, e aqui se destaca o fato de cerca de $40 \%$ da amostra não saber dizer o nome das medicações, indicando apenas por meio de imagens, ou seja, por meio da apresentação das fotos da medicação.

Os dados de retirada de medicação da farmácia (Tabela 1), por sua vez, indicam que quase $32 \%$ dos sujeitos não retirou a medicação no mês anterior à entrevista e que, em média, deixavam de retirar a medicação pelo menos 3 vezes ao ano. Também proveniente dos dados da farmácia, verificou-se que metade dos sujeitos fez menos do que $75 \%$ das retiradas esperadas de medicação, sendo que destes, aproximadamente $25 \%$ retirou menos da metade.

Os dados de exames laboratoriais se apresentaram satisfatórios, com média de CD4 acima de 400 cópias/ $\mathrm{mm}^{3}$ e carga viral indetectável em quase $70 \%$ dos casos. O CD4 apresentou correlação positiva com os dados de retirada de medicações da farmácia $(r=0,31 ; p=0,01)$, indicando que quanto mais medicações o sujeito retira,
Tabela 1. Distribuição da percentagem do total esperado de retiradas de medicações na farmácia em um ano

\begin{tabular}{lcc}
\hline Retiradas de medicação & $F$ & $\%$ \\
\hline $0 \%-25 \%$ & 9 & 12,3 \\
$26 \%-50 \%$ & 9 & 12,3 \\
$51 \%-75 \%$ & 19 & 26 \\
$76 \%-99 \%$ & 12 & 16,4 \\
$100 \%$ & 24 & 32,9 \\
\hline
\end{tabular}

melhor é a sua imunidade. Os valores de carga viral dos sujeitos com carga detectável, por sua vez, não apresentaram correlações significativas com outros indicadores de adesão. Dessa forma, pode-se afirmar que, no presente estudo, valores satisfatórios de CD4 e carga viral indetectável podem indicar melhor adesão ao tratamento.

Constatou-se, então, que a adesão aferida por meio de autorrelato tende a ser superestimada se comparada à mensurada nos registros da farmácia e até mesmo nos prontuários. No entanto, conforme descrito na literatura (Bonolo \& cols., 2007, Duong \& cols., 2001, Jordan \& cols., 2000), também podem ser indicadores confiáveis, o que acabou se confirmando no presente estudo, pois tanto a média de esquecimentos na última semana $(r=-0,26 ; p=0,05)$ quanto no último mês $(r=$ $0,24 ; p=0,05)$ se correlacionaram negativamente com a quantidade de retiradas de medicações da farmácia. Dessa forma, ao associar-se mais de um indicador para tentar aferir adesão, percebeu-se que, neste estudo, a quantidade de medicações retiradas na farmácia no período de 12 meses pode ser considerada o melhor e mais preciso indicador de falhas na adesão à TARV.

De acordo com os resultados apontados por esse último indicador, estima-se que a adesão média da amostra deste estudo à TARV tenha estado entre 50 e $75 \%$, o que vai ao encontro dos resultados encontrados na literatura, chamando atenção para o fato de apenas aproximadamente $33 \%$ da amostra ter retirado $100 \%$ da quantidade de doses esperadas, pois a maioria dos estudos estipula uma adesão adequada se tomadas entre 80 e 100\% das doses prescritas (Bonolo \& cols., 2007, Duong \& cols., 2001, Jordan \& cols., 2000).

Os dados de saúde mental, obtidos por meio do QSG (Tabela 2), indicam que cerca de 30\% a 35\% da amostra apresenta perfil sintomático para transtorno mental, com destaque para o fator "Distúrbios do Sono", que indicou cerca de $40 \%$ de sintomáticos mais 
$12 \%$ de limítrofes. Embora não tenham sido obtidos dados de outros estudos com o QSG para população com HIV e aids, esses índices vão ao encontro dos descritos por Sadock e Sadock (2007), e Straub (2005) sobre prevalência de transtornos mentais comuns nessa população. Com relação ao índice de distúrbios do sono, pode-se pensar na própria doença ou tratamento estarem interferindo ou provocando esse tipo de alteração, por ter esta condição se mostrado tão frequente. Outra explicação possível seriam os "distúrbios do sono" como sintomas de possíveis transtornos mentais comuns, pois cerca de $40 \%$ da amostra apresentou perfil sintomático para transtorno mental.
Feitas as caracterizações em termos de adesão e saúde mental, buscou-se correlacionar as variáveis desses dois aspectos da amostra, tendo em vista alcançar os objetivos do estudo, ou seja, verificar a influência de indicativos de transtornos mentais na adesão ao tratamento. A principal correlação encontrada entre saúde mental e adesão foi a correlação negativa entre desejo de morte e a quantidade de retirada de medicações da farmácia $(r=-0,27 ; p=0,05)$, indicando que os sujeitos menos aderentes apresentam maiores escores indicando desejo de morte. Os dados demonstram, inclusive, que esse desejo é mais acentuado em sujeitos mais novos, o que nos leva a perguntar se a não adesão

Tabela 2. Perfis sintomáticos dos fatores do QSG

\begin{tabular}{lccc}
\hline Fatores do QSG & $\begin{array}{c}\text { Sintomático (percentil } \\
>\text { ou igual a 90) }\end{array}$ & $\begin{array}{c}\text { Limítrofe (percentil } \\
\text { entre } 85 \text { e } 90)\end{array}$ & $\begin{array}{c}\text { Não sintomático } \\
\text { (percentil <85) }\end{array}$ \\
\hline Stress psíquico & $35,6 \%(\mathrm{n}=26)$ & $2,7 \%(\mathrm{n}=2)$ & $61,6 \%(\mathrm{n}=45)$ \\
Desejo de morte & $31,5 \%(\mathrm{n}=23)$ & - & $68,5 \%(\mathrm{n}=50)$ \\
Desconfiança no próprio desempenho & $28,8 \%(\mathrm{n}=21)$ & $9,6 \%(\mathrm{n}=7)$ & $61,6 \%(\mathrm{n}=45)$ \\
Distúrbios do sono & $38,4 \%(\mathrm{n}=28)$ & $12,3 \%(\mathrm{n}=9)$ & $49,3 \%(\mathrm{n}=36)$ \\
Distúrbios psicossomáticos & $32,9 \%(\mathrm{n}=24)$ & $1,4 \%(\mathrm{n}=1)$ & $65,8 \%(\mathrm{n}=48)$ \\
Saúde geral & $38,4 \%(\mathrm{n}=28)$ & $2,7 \%(\mathrm{n}=2)$ & $58,9 \%(\mathrm{n}=43)$ \\
\hline
\end{tabular}

não seria justamente uma tentativa de realização desse desejo. Por outro lado, é preciso lembrar que o desejo de morte aparece em muitos casos de sintomatologia depressiva, transtorno bastante frequente em pessoas que vivem com HIV e aids, dado que também aparece indicado em estudos que visavam investigar a ideação suicida em pacientes com HIV, mostrando que os indícios de ideação estão fortemente correlacionados com escores de depressão (Brian \& cols., 1998; Seidl \& cols., 2007).

Em termos dos demais fatores associados à adesão, destaca-se a percepção de suporte familiar, pois quase todos os fatores do IPSF se correlacionaram positivamente com a quantidade de medicação retirada da farmácia (fator afetivo-consistente $r=0,38, p=0,01$; fator adaptação familiar $r=0,31, p=0,01$; e fator geral $r=0,38, p=0,01)$, indicando que quanto mais percepção de suporte familiar adequado o sujeito tiver, maior será a adesão. Esses resultados corroboram os de outros estudos, como os realizados por Bonolo e colaboradores (2007), Calvetti (2010), Gordillo e colaboradores
(1999), Seidl e colaboradores (2007), enfatizando a importância dos aspectos anteriormente mencionados no fenômeno da adesão.

Foram feitas também análises de regressão para verificar se e quais variáveis relacionadas com saúde mental (fatores do QSG) e suporte familiar (fatores do IPSF) predizem a adesão (quantidade de medicação retirada). Primeiramente, pelo método FORWARD, $\operatorname{com} p=0,05$, que apontou para o IPSF (Total) explicando aproximadamente $15 \%$ da variância da variável adesão. Em seguida, foi feita a mesma análise com o método ENTER, e a variância explicada aumentou para $29 \%$, contudo ocorreu o problema da multicolinearidade (fator 1, 2 e total do IPSF). Por isso, optou-se por uma nova análise sem as variáveis que estavam muito relacionadas (retirou-se os fatores 1,2 e 3 do IPSF e manteve-se apenas o IPSF Total), utilizando o método ENTER, e a variância explicada foi de $28 \%$, não havendo multicolinearidade. Dessa forma, os resultados indicaram que o valor total do IPSF, prediz por si só, $15 \%$ da variância da adesão, e 
que as variáveis IPSF e QSG, juntas, predizem 28\%. Embora sejam índices não muito altos, são preditores e conferem ainda mais significado às correlações anteriores.

As demais correlações encontradas não dizem respeito à adesão, mas podem interferir indiretamente nesta e por isso merecem ser também apresentadas. A variável idade, por exemplo, destacou-se entre as sociodemográficas. Observou-se que sujeitos mais velhos tendem a apresentar maior percepção de suporte familiar $(r=0,32 ; p=0,01)$ e menos efeitos colaterais da TARV $(r=-0,28 ; p=0,05)$. Com relação aos dados clínicos, obtiveram-se correlações negativas significativas entre carga viral e os fatores stress psíquico $(r=-0,52$; $p=0,05)$ e distúrbios do sono $(r=-0,54 ; p=0,05)$ do QSG, indicando que indivíduos com carga viral detectável mais alta apresentam menos alterações mentais, o que certamente contraria a tese principal deste estudo, pois se sabe que sujeitos com carga viral alta provavelmente não aderem à TARV, ou seja, provavelmente esse achado se refere a indivíduos não aderentes. Pode-se pensar no tratamento como um causador de estresse, pois se sabe que este também pode interferir negativamente na qualidade de vida das pessoas vivendo com HIV e aids, quando apresentam, por exemplo, os diversos efeitos colaterais de curto e longo prazo da TARV (Brasil, 2008; Jordan \& cols., 2000; Sadock \& Sadock, 2007; Straub, 2005).

O CD4, por sua vez, apresentou correlação negativa com o número de doenças oportunistas $(r=-0,28$; $p=0,05)$ registradas no prontuário, ou seja, quanto maior o CD4, melhor a imunidade do indivíduo e, consequentemente, menor a quantidade de manifestações de doenças oportunistas. Por fim, destacaram-se as diversas correlações significativas dos fatores do IPSF com os fatores do QSG (Tabelas 3, 4 e 5).

Observam-se correlações negativas entre os fatores do IPSF e os do QSG, indicando que quanto maior a percepção de suporte familiar, menores os escores em boa parte dos fatores indicativos de transtornos mentais. Essas associações também implicam dizer que o sofrimento mental pode ser evitado ou diminuído pelo suporte familiar (Baptista, 2007; Oliveira \& Sommermam, 2008; Teodoro, 2012). No caso deste trabalho, os sujeitos foram abordados e entrevistados enquanto acometidos por um processo de doença e seu tratamento. Sendo assim, evidencia-se que quando a família vai ao encontro das necessidades de suporte do sujeito, esse suporte familiar pode servir como atenuador em termos de saúde mental.
Tabela 3. Correlações do fator 1 (afetivo-consistente) do IPSF com fatores do QSG

\begin{tabular}{lcc}
\hline F1 (IPSF) versus & $r$ & $p$ \\
\hline Desejo de morte (QSG) & $-0,29$ & 0,05 \\
Distúrbios psicossomáticos (QSG) & $-0,25$ & 0,05 \\
\hline
\end{tabular}

Tabela 4. Correlações do fator 2 (adaptação familiar) do IPSF com fatores do QSG

\begin{tabular}{lcc}
\hline F2 (IPSF) versus & $r$ & $p$ \\
\hline Stress psíquico (QSG) & $-0,38$ & 0,01 \\
Desejo de morte (QSG) & $-0,34$ & 0,01 \\
Distúrbios do sono (QSG) & $-0,31$ & 0,01 \\
Distúrbios psicossomáticos (QSG) & $-0,37$ & 0,01 \\
Saúde geral (QSG) & $-0,33$ & 0,01 \\
\hline
\end{tabular}

Tabela 5. Correlações do fator 3 (autonomia familiar) do IPSF com fatores do QSG

\begin{tabular}{lcc}
\hline F3 (IPSF) versus & $r$ & $p$ \\
\hline Stress psíquico (QSG) & $-0,34$ & 0,01 \\
Desejo de morte (QSG) & $-0,31$ & 0,01 \\
Distúrbios do sono (QSG) & $-0,39$ & 0,01 \\
Distúrbios psicossomáticos (QSG) & $-0,32$ & 0,01 \\
Saúde geral (QSG) & $-0,32$ & 0,01 \\
\hline
\end{tabular}

\section{Considerações finais}

O estudo delineado propôs-se avaliar a adesão à TARV em pacientes com HIV e aids e buscar associação desta com as variáveis percepção de suporte familiar e indicativos de transtornos mentais. De forma geral, esse propósito foi satisfeito e foram obtidos resultados bastante significativos. A adesão foi aferida por mais de uma medida de avaliação, autorreferidas ou não, que se correlacionaram entre si. Foi obtida uma estimativa de que a amostra tome, em média, entre 50 e $75 \%$ das drogas prescritas, e o registro de retirada de medicações da farmácia foi considerado o indicador de adesão mais preciso.

Em termos de saúde mental, os resultados do QSG indicaram cerca de $35 \%$ de perfis sintomáticos para transtornos mentais na amostra. Portanto, foram 
observados índices significativos tanto para adesão irregular à TARV, quanto para saúde mental, embora essas duas variáveis não necessariamente estejam correlacionadas. Somente um fator do QSG obteve correlação direta com adesão, e este se refere a "desejo de morte", possivelmente relacionado à depressão, transtorno mais frequente nessa população.

A percepção de suporte familiar mostrou-se a variável com maior número de correlações significativas, tanto com a adesão quanto com a saúde mental. Quase todos os fatores do IPSF correlacionaram-se positivamente com a adesão e negativamente com a maioria dos fatores do QSG, apontando o suporte familiar como um fator facilitador da adesão ao tratamento e atenuador em termos de saúde mental no contexto $\mathrm{HIV} /$ aids.

A avaliação psicológica em ambientes médicos pode ser considerada uma adequada ferramenta na tomada de decisões sobre o diagnóstico, tratamento e adesão a este. Levando-se os dados apresentados no estudo em consideração e a importância e atualidade da temática para a avaliação psicológica, especialmente no que se refere ao contexto da saúde pública, sugere-se a realização de outros estudos com amostras maiores e mais representativas, com a perspectiva de encontrar outras variáveis, além daquelas aqui consideradas.

Considerando que neste estudo foi avaliada especificamente a percepção de suporte familiar, para pesquisas futuras propõem-se avaliar outros tipos de suporte (social, por exemplo), visando um conhecimento mais abrangente do modo como os diferentes tipos de suporte ou apoio recebidos ou percebidos interferem positiva ou negativamente em contextos da saúde e doença. Com relação à saúde mental, foram avaliados por meio do QSG apenas indicativos ou sintomas em saúde mental, não tendo sido feitos diagnósticos de qualquer espécie. Para futuras pesquisas, seria interessante investigar diagnósticos específicos em saúde mental como, por exemplo, a depressão, transtorno de humor descrito como o mais frequente no contexto estudado; ou ainda, a avaliação de fatores de personalidade individuais possivelmente associados às condutas investigadas.

\section{Referências}

Adão, V. M., \& Caraciolo, J. M. M. (2007). Fatores que influenciam a adesão aos antirretrovirais. Em J. M. M. Caraciol, \& E. Shimma. (Eds.), Adesão da teoria à prática: experiências bem sucedidas no Estado de São
Paulo. (pp. 28-42). São Paulo: Centro de Referência e Treinamento DST e aids.

Ammassari, A., Antinori, A., Aloisi, M. S, Trotta, M. P, Murri, R., Bartoli, L., Monforte, A. A, Wu, A. W., \& Starace, F. (2004). Depressive symptoms, neurocognitive impairment, and adherence to highly active antiretroviral therapy among HIV-infected persons. Psychosomatics, 45(5), 394-402. Disponível em: http://psy.psychiatryonline.org/cgi/content/ abstract/45/5/394

Baptista, M. N. (2007). Inventário de Percepção de Suporte Familiar (IPSF): estudo componencial em duas configurações. Psicologia Ciência e Profissão, 27(3), 496-509. Disponível em: http://pepsic. bvs-psi.org.br/scielo.php?script=sci_arttext\&pi$\mathrm{d}=$ S1414-98932007000300010\&lng $=$ pt\&nrm $=$ iso

Baptista, M. N. (2010). Inventário de Percepção de Suporte Familiar - IPSF. São Paulo: Vetor.

Bonolo, P. de F., Gomes, R. R. de., \& Guimarães, M. D. C. (2007). Adesão à terapia anti-retroviral (HIV/aids): fatores associados e medidas de adesão. Epidemiol. Serv. Saúde, 16(4), 261-78. Disponível em: http://scielo.iec.pa.gov. br/scielo.php? script $=$ sci_pd f\&pid $=$ S167949742007000400005\&lng=pt\&nrm $=$ iso\&tlng $=$ pt

Brasil. Ministério da Saúde. (2007). Programa Nacional de DST-AIDS. Disponível em http://www.aids.gov.br

Brasil. Ministério da Saúde. Programa Nacional de DST-AIDS. (2008). Manual de adesão ao tratamento para pessoas vivendo com HIV e aids.

Brasil. Ministério da Saúde. Boletim epidemiológico aids. DST. (2011). Disponível em http://www.aids.gov.br/ publicacao/2011/boletim_epidemiologico_2011.

Brian, K. B, Raphael, B., Judd, F., Perdices, M., Kernutt, G., Burnett, P., Dunne, M., \& Burrows, G. (1998). Suicidal ideation, suicide attempts, and HIV Infection. Psychosomatics, 39(5), 405-15. Disponível em: http:/ / eprints.qut.edu.au/27121/1/Kelly_Raphael_Judd1998b.pdf

Calvetti, P. U. (2010). Fatores biopsicossociais preditivos para a adesão e qualidade de vida em pessoas que vivem com HIV/Aids bem-sucedidas no tratamento de Saúde. Tese de Doutorado. Disponível em: http://www3.crt. saude.sp.gov.br/arquivos/arquivos_biblioteca_ crt/PRISLA_UCKER_CALVETTI.pdf. 
Campos, L. N., Guimarães, M. D. C, \& Remien, R. H. (2010). Anxiety and depression symptoms as risk factors for non-adherence to antiretroviral therapy in Brazil. AIDS Behav., 14, 289-299. Disponível em: http://www.springerlink.com/content/ h147700717257307/

Caraciolo, J. M. M. (2007). Adesão aos antirretrovirais. Em J. M. M. Caraciolo, \& E. Shimma (Eds). Adesão da teoria à prática: experiências bem sucedidas no Estado de São Paulo. (pp. 10-26). São Paulo: Centro de Referência e Treinamento DST e aids.

Cozby, P. C. (2003). Métodos de pesquisa em ciências do comportamento. São Paulo: Atlas.

Dancey, C. P., \& Reidy, J. (2006). Estatística sem matemática para psicologia. 3a ed. Porto Alegre: Artmed.

Dourado, I., Veras, M. A. de S., Barreira, D., \& Brito, A. $M$ de. (2006). Tendências de epidemia de Aids no Brasil após a terapia anti-retroviral. Rev Saúde Pública, 40(Supl), 9-17. Disponível em: http://www. scielo.br/pdf/rsp/v40s0/03.pdf.

Duong, M., Piroth, L., Grappin, M., Forte, F., Peytavin, G., \& Buisson. M. (2001). Evaluation of the patient medication adherence questionnaire as a tool for self-reported adherence assessment in HIVinfected patients on antiretroviral regimens. HIV Clinical Trials, 2(2), 128-35. Disponível em: http:// www.ncbi.nlm.nih.gov/pubmed/11590521

Giovelli, G. R. M. (2009). Relação entre sintomas de depressão, suporte social, qualidade de vida e adesão ao tratamento em pessoas que vivem com HIV/aids. Dissertação de Mestrado. PUC-RS. Disponível em: http://biblioteca.universia.net/html_bura/ficha/ params $/$ title $/$ rela $\% \mathrm{C} 3 \% \mathrm{~A} 7 \% \mathrm{C} 3 \% \mathrm{~A} 3 \mathrm{O}$-sintomasdepress $\% \mathrm{C} 3 \% \mathrm{~A} 3 \mathrm{O}$-suporte-social-qualidade-vida-ades \%C3\%A3o-ao-tratamento/id/42260428. html.

Gir, E.,Vaichulonis, C. G, \& Oliveira, M. D. (2005). Adesão à terapêutica anti-retroviral por indivíduos com HIV/AIDS assistidos em uma instituição do interior paulista. Rev. Latino-Am. Enfermagem, 13(5), 634-41. Disponível em: http://www.scielo.br/ pdf/rlae/v13n5/v13n5a05.pdf.

Gordillo, V., Del Amo, J., Soriano, V., \& González-Lahoz, J. (1999). Sociodemographic and psychological variables influencing adherence to antiretroviral therapy. AIDS, 13, 1763-1769. Disponível em: http://www.aidsonline.com/pt/ re/aids/abstract.00002030-199909100-00021. htm; jessionid = L6CLyWzCnc9nrJ Qn6cLnfg6j6kG81dgvgGMs3fC3PhnMlZzlFNMF!231517226!181195629!8091!-1

Jordan, M. S., Lopes, J. F., Okazaki, E., Komatsu, C. L., \& Nemes, M. I. B. (2000). Adesão ao tratamento anti-retroviral em aids: revisão da literatura médica. Em P.R. Teixeira, V. Paiva, \& E. Shimma. (Orgs.), Tá difícil de engolir? experiências de adesão ao tratamento anti-retroviral em São Paulo. (pp. 5-25). São Paulo: Nepaids.

Lewi, D. A., Turcato Jr., G., Tenore, S., Ferreira, P. R. A., Castelo Filho, A., \& Diaz, R. S. (2007). Síndrome da imunodeficiência adquirida. Em F. C. do Prado, J. Ramos \& J. R. do Valle (Orgs.), Atualização terapêutica. (pp. 867- 872). 23ª . ed. São Paulo: Artes Médicas.

Lignani Júnior, L., Greco, D. B., \& Carneiro, M. (2001). Avaliação da adesão aos anti-retrovirais em pacientes com infecção pelo HIV/AIDS. Rev. Saúde Pública, 35(6), 495-501. Disponível em: http:// www.scielosp.org/pdf/rsp/v35n6/7060.pdf.

Mello, V. A., \& Malbergier, A. (2006). Depression in women infected with HIV. Rev. Bras. Psiquiatr., 28(1), 10-17. Disponível em: http://www.scielo.br/scielo. php?pid=S1516-44462006000100004\&script $=$ sci_arttext.

Moraes, M. J de., Oliveira, A. C. P de., \& Tostes, M de A. (2006). AIDS e psiquiatria. Em N. J. Botega (Org.), Prática psiquiátrica no hospital geral: interconsulta e emergência. (pp. 373-394). 2a . ed. Porto Alegre: Artmed.

Oliveira, E. B. S. de.,\& Sommermam, R. D. G. (2008). A família hospitalizada. Em B. W. Romano (Org.), Manual de psicologia clínica para hospitais (pp. 117-143). São Paulo: Casa do Psicólogo.

Pasquali, L., Gouveia, V. V., Andriola, W. B, Miranda, F. J., \& Ramos, A. L. M. (1996). Questionário de Saúde Geral de Goldberg: manual técnico QSG. Adaptação brasileira. São Paulo: Casa do Psicólogo.

Rachid, M., \& Schechter, M. (2005). Manual de HIV/ AIDS. $8^{\text {a }}$ ed. Rio de Janeiro: Revinter.

Reis, A. C., Lencastrea, L., Guerra, M. P., \& Remorb, E. (2009). Relação entre sintomatologia psicopatológica, adesão ao tratamento e qualidade de vida na infecção HIV e AIDS. Psicologia: Reflexão e Crítica, 
23(3), 420-9. Disponível em: http://www.scielo. br/pdf/prc/v23n3/02.pdf.

Sadock, B. J., \& Sadock, V. A. (2007).Compêndio de psiquiatria: ciências do comportamento e psiquiatria clínica. $9^{a}$ ed. Porto Alegre: Artmed.

Seidl, E. M. F., Melchiades, A., Farias, V., \& Brito, A. (2007). Pessoas vivendo com HIV/AIDS: variáveis associadas à adesão ao tratamento anti-retroviral. CAD. Saúde Pública, 23(10), 2305-2316. Disponível em: http://www.scielosp.org/scielo. php?script $=$ sci_pdf\&pid $=$ S0102-311X200700100 0006\&lng $=$ en\&nrm $=$ iso\&tlng $=$ pt.

Straub, R. O. (2005). Psicologia da saúde. Porto Alegre: Artmed.

Teodoro, M. L. M. (2012). Alguns instrumentos para avaliação familiar no Brasil. Em M. N. Baptista, \& M. L. M. Teodoro (Orgs.), Psicologia de família: teoria, avaliação e intervenção. (pp.168-175). Porto Alegre: Artmed.
United Nations Programme on HIV/Aids. (UNAIDS). (2010). AIDS epidemicupdate. Disponível em: http:// www.unaids.org

Weiss, J. J., \& Bangsberg, D. R. (2008). Psychiatric aspects of adherence to medical care and treatment for HIV/AIDS. Em M. A. Cohen, \& J. M. Gorman (eds). (pp. 281-295). Comprehensive Textbook of AIDS Psychiatry. New York: Oxford University Press.

Yun, L. W. H, Maravi, M., Kobayashi, J. S, Barton, P. L, \& Davidson, A. J. (2005). Antidepressant treatment improves adherence to antiretroviral therapy among depressed HIV-infected patients. Acquir. ImmuneDefic. Syndr, 38(4), 432-438. Disponível em: http://www.jaids.org/ pt/re/jaids/abstract.00126334-200504010-00008. $\mathrm{htm}$; sessionid $=\mathrm{L} 6 \mathrm{GMJ} 8 \mathrm{DYKZS0} 5 \mathrm{nSNn}-$ JxK2RvX6htvnxcy3z4yJsDxQpZVtlWTTv7g!231517226!181195629!8091!-1

Recebido em: 30/04/2013 Reformulado em: 26/02/2014 Aprovado em: 13/05/2014

Sobre os autores:

Luiza Azem Camargo é mestre em Ciências pela Coordenadoria de Controle de Doenças da Secretaria de Estado da Saúde de SP, psicóloga do Serviço de Extensão ao Atendimento de Pacientes com HIV/Aids do HCFMUSP e membro do grupo de neurociências do Instituto de Infectologia Emílio Ribas. E-mail: luiza.azem@gmail.com

Cláudio Garcia Capitão é professor dos cursos de graduação e de Pós-Graduação Stricto Sensu em Psicologia da Universidade São Francisco, membro do grupo de neurociências do Instituto de Infectologia Emílio Ribas.

Elvira Maria Ventura Filipe é doutora em Psicologia da Saúde pela University of London, professora do curso de graduação da Universidade Nove de Julho e do Programa de Pós-Graduação em Ciências da Coordenadoria de Controle de Doenças da Secretaria de Estado da Saúde de SP. Foi diretora do Núcleo de Prevenção do Centro de Referência e Treinamento DST/AIDS - SP.

Endereço para correspondência:

Av. Dr. Arnaldo, 165, Cerqueira César, São Paulo-SP.

CEP: 01246-900

E-mal: luiza.azem@gmail.com 